\title{
An Efficient Zero-Skew Routing Algorithm
}

\author{
Masato Edahiro \\ C\&C Research Laboratories, NEC Corporation \\ Miyazaki, Miyamae-ku, Kawasaki 216, Japan \\ Department of Computer Science, Princeton University \\ Princeton, NJ 08544-2087, USA
}

\begin{abstract}
A bucket algorithm is proposed for zeroskew routing with linear time complexity on the average. Our algorithm is much simpler and more efficient than the best known algorithm which uses Delaunay triangulations for segments on Manhattan distance. Experimental results show that the linearity of our algorithm is accomplished. Our algorithm generates a zero-skew routing for 3000-pin benchmark data within 5 seconds on a 90MIPS RISC workstation.
\end{abstract}

\section{INTRODUCTION}

With scaling down the dimensions of devices, zero-skew clock routing technique becomes more critical for high performance VLSI's because wiring delay grows comparable to (or even dominates) gate switching delay. Even though exact zero skew was achieved [11], more efficient delay minimization algorithms are still desired.

There have been four types of algorithms proposed for the total-wire-length minimization in zero-skew routing. Matching-based [3] and partitioning-based algorithms [2] were the first practical algorithms for this problem whose time complexity is $O\left(n \log ^{2} n\right)$. A bottom-up construction algorithm [8] reduced the total wire length by $15 \%$, though its time complexity is $O\left(n^{2} \log n\right)$. A clustering-based algorithm [4] improved the time complexity to $O(n \log n)$ without any increase of the total wire length. Thus, the clustering-based algorithm is currently the most efficient in a theoretical sense. In practice, however, the clustering-based algorithm might not be efficient because it is required to construct Delaunay triangulations for segments on Manhattan distance, for which no practical algorithm has been proposed.

As for the total delay minimization, Edahiro [5] recently proposed delay time estimation and optimum wiresizing methods. He proposed a minimum-delay zero-skew routing algorithm by combining these methods with the clustering-based algorithm [4].

In this paper, we propose a zero-skew routing algorithm that is much simpler and practically more efficient than the clustering-based algorithm. The time complexity of our algorithm is linear on the average. In this algorithm, we introduce a relation graph to represent the nearness

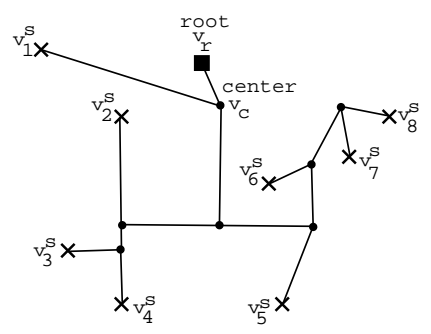

Figure 1: Clock Tree with a root $v_{r}$ and leaves $S=$ $\left\{v_{1}^{s}, v_{2}^{s}, \ldots, v_{8}^{s}\right\}$.

between nodes on clock trees. In addition, we define an independent edge set on the relation graph that is used to construct minimum-delay zero-skew routings in a bottomup fashion.

We use a bucket algorithm to construct relation graphs. The bucket algorithm finds 'nearness' between nodes in linear time on the average. In order to search the independent edge set, a depth first traverse is utilized on the relation graphs, which is performed in linear time. As a result, linearity of our algorithm is accomplished.

Experimental results show that, while the delay time increases $3 \%$ in our algorithm compared with the best known algorithm [4, 5], our algorithm is much simpler and more efficient. Our algorithm generates a zero-skew routing for 3000-pin benchmark data within 5 seconds on a 90MIPS RISC workstation.

\section{Zero-Skew Routing}

Given a fan-out terminal $v_{r}$ and a set of $n$ fan-in terminals $S=\left\{v_{1}^{s}, v_{2}^{s}, \ldots, v_{n}^{s}\right\}$, a clock tree is defined by a tree rooted by $v_{r}$ whose $n$ leaves are $S$ (Fig. 1 ). We call the fan-out terminal root and fan-in terminals leaves. A set of leaves in the subtree rooted by a node $v$ is called leaves connecting to $v$ and denoted by $S_{v}$. In this paper, clock trees are always binary, though nodes may degenerate and they do not look binary in some cases. Also, we call the nearest internal node to the root the center denoted by $v_{c}$.

We assume that the load capacitance $C\left(v_{i}^{s}\right)$ is given for each leaf $v_{i}^{s}$, which is usually the gate capacitance of transistors associated with the leaf. Also, the load capacitance $C(v)$ for an internal node $v$ is defined by the total capacitance in $S_{v}$ that includes wire capacitance as well as gate capacitance. 
Then, a zero-skew routing for the given root and leaves is defined by a clock tree in which all delay time from the root to all leaves is equal. From this definition, it is clear that, for any node in the zero-skew routing, all delay from the node $v$ to leaves in $S_{v}$ should be equal, which is called delay time $\tau(v)$ for $v$. For leaves $v_{i}^{s}, \tau\left(v_{i}^{s}\right)=0$.

An exact zero-skew routing can be constructed in a bottom-up fashion by repeatedly calculating the position of an internal node $v$ from the positions of children $v_{1}$ and $v_{2}$ of $v$ using the following equations derived from $\pi$-model [5]:

$$
\begin{aligned}
\tau(v) & =\frac{r l_{1}}{w_{1}}\left(\frac{c l_{1} w_{1}}{2}+C\left(v_{1}\right)\right)+\tau\left(v_{1}\right) \\
& =\frac{r l_{2}}{w_{2}}\left(\frac{c l_{2} w_{2}}{2}+C\left(v_{2}\right)\right)+\tau\left(v_{2}\right), \\
C(v) & =C\left(v_{1}\right)+C\left(v_{2}\right)+c\left(l_{1} w_{1}+l_{2} w_{2}\right),
\end{aligned}
$$

where $l_{1}\left(l_{2}\right)$ and $w_{1}\left(w_{2}\right)$ are length and width of the wire from $v$ to $v_{1}\left(v_{2}\right)$, and $r$ and $c$ are wire resistance and capacitance for an unit length and width wire.

This operation to determine the location of a node $v$ is called the zero-skew merge. At a zero-skew merge, it is clear that $l_{1}+l_{2} \geq l$, where $l$ is the distance between $v_{1}$ and $v_{2}$. Therefore, in order to minimize the delay time, it is desired to find $v$ such that $l_{1}+l_{2}=l$. If there is no such a $v$, zero-skew routing algorithms should use a detour. Although the number of detours depends on the algorithms and the input data, detours hardly appear in actual layouts on 'good' algorithms.

Note that, in Manhattan distance, a set of feasible points for $v$ satisfying the above equations forms a diagonal segment in general. This segment (or point for a leaf) is called the segment for $v$ or simply $v$. The segment for $v$ can be calculated in constant time even if children are also expressed by diagonal segments $[2,6]$.

\section{DEFinitions}

In this section, we define the relation graph and the independent edge set on the relation graph, which play important roles in our algorithm.

\section{A. Relation Graph}

First, we define the relation graph with a relation function $f\left(v_{i}, v_{j}\right)$ of segments $v_{i}$ and $v_{j}$. Examples of relation functions will be described later. Let $K$ be a set of segments. The relation graph $G(K, E)$ for $K$ is defined by the weighted directed graph such that

i) Each node $v \in K$ has exact one out-going edge, so that $|E|=|K|$

ii) If $\left(v_{i}, v_{j}\right) \in E$ for $v_{i}, v_{j} \in K, \forall v_{k} \in K-\left\{v_{i}\right\}$, $f\left(v_{i}, v_{j}\right) \leq f\left(v_{i}, v_{k}\right)$ (if there is a tie, one of the tie edges is arbitrarily selected),

iii) Each edge $e=\left(v_{i}, v_{j}\right) \in E$ has a weight $w(e)=$ $f\left(v_{i}, v_{j}\right)$.

An example of relation graph is depicted in Fig. 2. As we explain in the following sections, some edges in relation graphs are selected at each step of our algorithm, and,

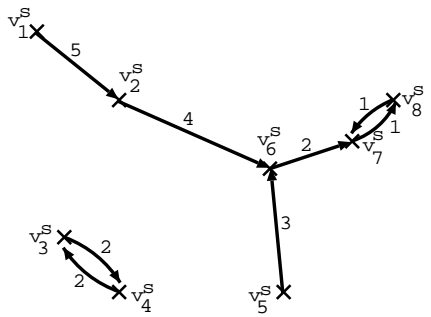

Figure 2: Relation Graph for $K=\left\{v_{1}^{s}, \ldots, v_{8}^{s}\right\}$ in Fig. 1.

for each selected edge, its two endpoints are zero-skew merged. We say that the zero-skew merge is associated with the edge.

We use two types of relation function. The nearestneighbor relation function calculates the Manhattan distance between segments for $v_{i}$ and $v_{j}$. Note that the relation graph with this function is equivalent to the nearest neighbor graph $[4,9]$. Since the distance between segments for $v_{i}$ and $v_{j}$ turns out the increase of the total wire length when $v_{i}$ and $v_{j}$ are zero-skew merged, the nearest neighbor relation tries to minimize the total wire length.

The other is called the minimum-delay relation function. Since the total delay $t_{d}$ is well-estimated by the following formula [5]:

$$
t_{d} \approx 1.85 \frac{C\left(v_{r}\right)}{\beta V_{D D}}+0.7 \tau\left(v_{r}\right)
$$

where $\beta$ is the MOS transistor gain factor, we define the minimum-delay relation function by:

$$
f\left(v_{i}, v_{j}\right)=1.85 \frac{C(v)}{\beta V_{D D}}+0.7 \tau(v),
$$

where $v$ is the segment obtained by the zero-skew merge between $v_{i}$ and $v_{j}$. It is clear that this relation function tries to minimize the total delay. Note that it is not difficult that the minimum-delay relation function includes the wire width optimization technique proposed in [5].

In this paper, we analyze properties of the relation graphs and our routing algorithm only for the nearestneighbor relation function because the minimum-delay relation function is too complicated to look into. Fortunately, since short wire length tends to cause short delay time, these two relation functions have similar behavior in zero-skew routing algorithms.

Now, we show a property for the nearest neighbor graph [4]. Let the sequence $\left\{e_{0}, e_{1}, \ldots, e_{|K|-1}\right\}$ be the edges in $E$ sorted by their weights in non-decreasing order. Then, the following property characterizes the weight of $e_{i}$.

Property 1 For $0 \leq \forall i<|K|-1$,

$$
w\left(e_{i}\right) \leq \frac{D}{\sqrt{|K|-i}-1},
$$

where $D$ is the diameter of $K$.

\section{B. Independent Edge Set}

In a relation graph, two edges are called dependent if these edges share an endpoint. Also, an edge set is called 


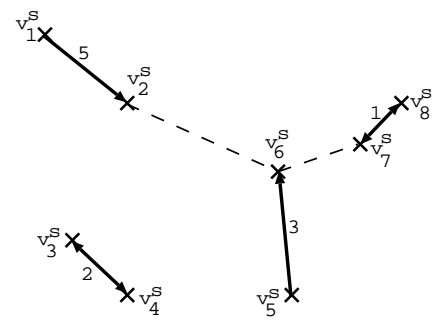

Figure 3: Independent Edge Set for Fig. 2 (solid lines).

independent if no two edges in the set are dependent. An example of the independent edge set is shown in Fig. 3.

Since zero-skew merges associated with dependent edges cannot be implemented at the same time, we need to select an independent edge set from a relation graph to minimize the total wire length or the total delay. We describe the selection algorithm in the next section.

\section{Algorithm}

In this section, we propose a zero-skew routing algorithm with linear time complexity on the average. In our algorithm, we use a parameter $k>1$. Typically, $2 \leq k \leq 5$. Also, we use a function $s(a, b, c)$ where $a \leq c$ defined by

$$
s(a, b, c)= \begin{cases}a ; & a \geq b, \\ b ; & a<b<c \\ c ; & b \geq c\end{cases}
$$

First, we outline our algorithm. Let $K$ be a subset of nodes in the zero-skew routing we are constructing. Initially, $K \equiv S$. At each step of our algorithm, after constructing a relation graph for $K$, an independent edge set on the relation graph is selected. Then, zero-skew merges between $v_{i}$ and $v_{j}$ are applied for all edges $\left(v_{i}, v_{j}\right)$ in the set, and $K$ is updated by deleting $v_{i}$ and $v_{j}$ and inserting $v$ that is generated by the zero-skew merge associated with $\left(v_{i}, v_{j}\right)$. Repeating this procedure several times, the position of the center $v_{c}$ is determined.

After that, a clock routing is generated by embedding wires in a top-down fashion using positions calculated above.

It is important to note that, as discussed in [4], applying zero-skew merges associated with all edges in an independent edge set could cause longer total wire length and/or total delay time. They pointed out that it is a better idea to limit only $|K| / k$ smallest-weight edges in the relation graph. This technique is also implemented in our algorithm.

Let us take a simple example in Fig. 4. We assume $k=2$. For a set of leaves $S$ (Fig. 4 (a)), the relation graph is depicted in Fig. 4 (b), in which weight $w(e)$ is attatched on each edge $e$. An independent edge set is shown in Fig. 4 (c). Zero-skew merges are implemented for two edges in the independent set because of the limit $k=2$. The new segments are $v_{9}$ from $\left(v_{3}^{s}, v_{4}^{s}\right)$ and $v_{10}$ from $\left(v_{7}^{s}, v_{8}^{s}\right)$ (Fig. 4 (d)). $K$ is updated to $\left\{v_{1}^{s}, v_{2}^{s}, v_{5}^{s}, v_{6}^{s}, v_{9}, v_{10}\right\}$, and $v_{9}\left(v_{10}\right)$ becomes a father of $v_{3}^{s}$ and $v_{4}^{s}\left(v_{7}^{s}\right.$ and $\left.v_{8}^{s}\right)$ in the zeroskew routing we are constructing (Fig. 4 (e)). Then, a relation graph is reconstructed for the updated $K$ (Fig. 4 (f)). After repeating this operation, the segment for $v_{c}$ is obtained. Figure $4(\mathrm{~g})$ shows all calculated segments, and their tree structure is depicted in Fig. $4(\mathrm{~h})$.

Then, we first draw a wire from $v_{r}$ to the nearest position on the segment for $v_{c}$. Drawing all wires from father to children on the clock tree in a top-down fashion, a zero-skew routing is generated (Fig. 4 (i)).

In the following sections, we describe our algorithm in detail.

\section{A. Relation Graph Construction}

In the algorithm proposed in [4], the nearest neighbor graph is constructed from a Delaunay triangulation [9]. However, calculating Delaunay triangulations seems expensive in practice. In this section, we propose a new construction method using the bucketing technique, which accomplishes linear time complexity on the average.

\section{(1) Bucket Decomposition}

Buckets are spatial partitioning by meshes. In our algorithm, all segments in a node set $K$ are distributed in $\Theta(|K|)$ buckets. Since we need to store diagonal segments in buckets, we use buckets partitioned by diagonal lines in our implementation. An example of the bucket decomposition for Fig. 4 (a) is shown in Fig. 5.

The bucket size is determined by the following way. Let $D$ be the diameter of $K$, that is, the distance between the farthest pair of elements in $K$. Also, let $d=\sqrt{s(2,(1-1 / k)|K|+1,|K|)}-1$. Then, the size of a bucket is calculated by $(D / d) \times(D / d)$.

There are two significant characteristics in this partitioning method. First, on this partition, we have only to check nine buckets for a portion of each segment $v_{i}$ inside a bucket to find an edge $\left(v_{i}, v_{j}\right)$ that is possible to belong to $|K| / k$ smallest-weight edges in the nearest neighbor graph. This is simply proven. By Property 1 , for the $(|K| / k)$-th smallest-weight edge $e$ in the nearest neighbor graph, the upper bound of $w(e)$ is $D / d$. This means that we do not need to check any segment whose distance from $v_{i}$ is more than $D / d$. Since the bucket size is $(D / d) \times(D / d)$, for any portion of segment inside a bucket, we have only to check nine buckets.

The second characteristic is that the number of buckets is $\Theta(|K|)$. This is also proven easily. From the definition of buckets, the number of buckets is $d^{2}$, which is $\Theta(|K|)$.

Now, we assume that segments in $K$ are uniformly distributed, and that length of any segment in $K$ is not very long so that each segment intersects with $O(1)$ buckets. Note that these assumptions are satisfied for benchmark data $[7,11]$. Under these assumptions, it is easy to see that each bucket contains $O(1)$ segments in $K$ on the average, and it requires only $O(1)$ time for a segment $v_{i}$ in $K$ to find an edge $\left(v_{i}, v_{j}\right)$ that is possible to belong to $|K| / k$ smallest-weight edges in the nearest neighbor graph.

\section{(2) Relation Graph Construction}

Next, a relation graph is constructed using the bucket decomposition described above. In our algorithm, however, we do not construct an entire relation graph, but generate its subgraph that includes at least $|K| / k$ smallest-weight edges in the nearest neighbor graph. This 


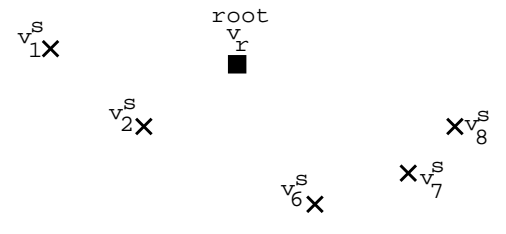

$v_{3}^{s} x$

$\mathrm{x}_{4}^{\mathrm{s}} \quad \mathrm{v}_{5}^{\mathrm{s}} \mathrm{X}$

(a)

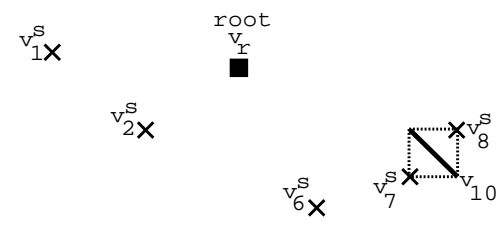

${ }_{v_{9}}^{v_{3}^{s} x} /_{v_{5}^{s}}^{s}$

(d)

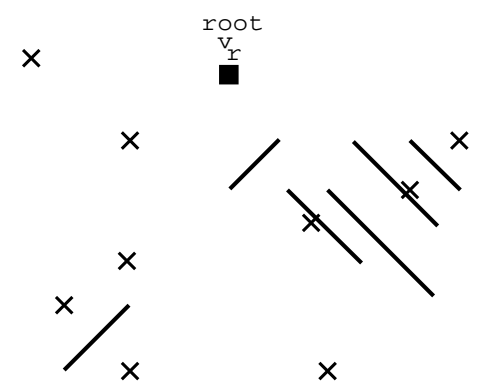

(g)

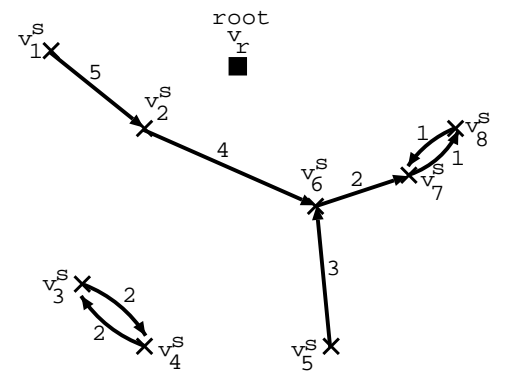

(b)
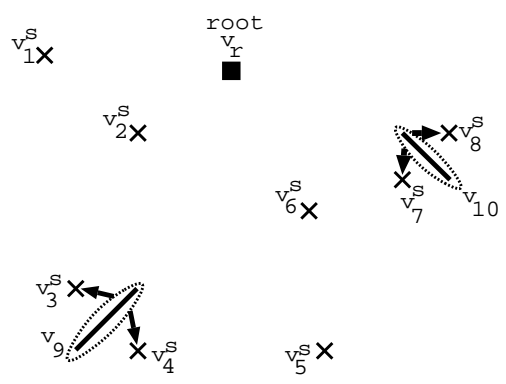

(e)

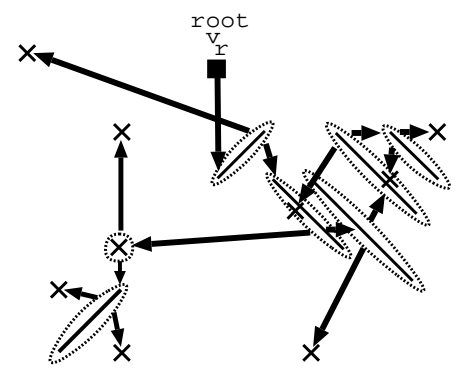

(h)

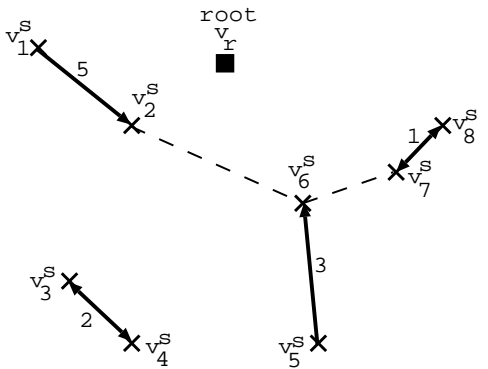

(c)

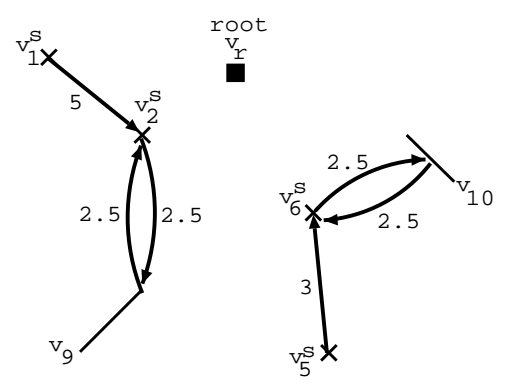

(f)

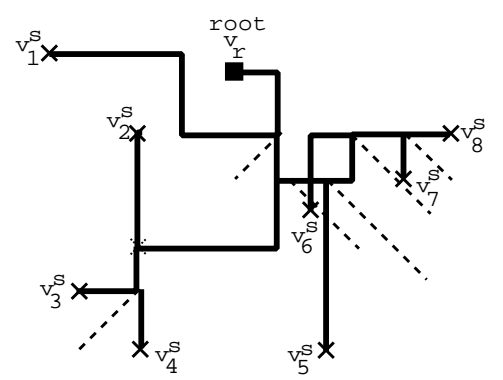

(i)

Figure 4: Example of Our Algorithm.

subgraph is sufficient for our purpose because only $|K| / k$ smallest-weight edges are necessary in our algorithm as we have discussed above.

In order to calculate the subgraph, we first construct the bucket decomposition. Then, for each segment $v_{i} \in$ $K$, we find a segment $v_{j}$, which minimizes the relation function $f\left(v_{i}, v_{j}\right)$ among all segments in $K$ contained in nine buckets around $v_{i}$. From the above discussion, the following property is clear.

Property 2 Our bucket algorithm requires $O(|K|)$ time on the average to construct a subgraph of the nearest neighbor graph for $K$, which includes at least $|K| / k$ smallest-weight edges in the nearest neighbor graph.

\section{B. Independent Edge Set Selection}

In this section, we describe a linear-time algorithm to select an independent edge set from a relation graph. It is desired that edge weights in the independent set are as small as possible. First, we describe a simple algorithm whose complexity is not linear.

\section{(1) Basic Algorithm}

In this algorithm, we first sort all edges in the relation graph for $K$ in non-decreasing order. Also, let $I$ be a set of edges and initially $I=\phi$. Then, edges are taken one by one in the sorted order. If an edge is independent from all edges in $I$, add it to $I$; and otherwise, the edge is just discarded. After checking $|K| / k$ smallest-weight edges, an independent edge set has been stored in $I$. We call the independent edge set by this algorithm the canonical independent set. Fig. 4 (c) is the canonical independent set on the relation graph in Fig. 4 (b) for $K$.

Note that the time complexity for this algorithm is $O(|K| \log |K|)$ for sorting.

\section{(2) Linear Time Algorithm}

Next, we propose a linear time algorithm to find the canonical independent set. Before explaining our algorithm, we state the condition for the canonical independent set. 
Property 3 Edge $e=\left(v_{i}, v_{j}\right)$ is in the canonical independent set if no edge $e^{\prime}$ of $w\left(e^{\prime}\right) \leq w(e)$ incident to $v_{i}$ or $v_{j}$ is in the canonical independent set.

Now, we propose our algorithm to find the canonical independent set. Our algorithm uses a depth first search. In our algorithm, each edge $e$ has a flag flag(e) initialized by $U N S E E N$. If an edge $e$ is to be in the canonical independent set, flag(e) is set to $Y E S$, and if not, flag(e) is set to $N O$.

\section{Algorithm Find_Independent_Set}

Step 1: For all $v \in K$, check_dependency $(v, N U L L)$.

Step 2: Canonical Independent Set $:=\{$ All edges with $Y E S$ flag\}.

procedure check_dependency $(v, e 0)$

Step 1: If $e 0 \neq N U L L$ AND flag $(e 0) \neq U N S E E N$, return.

Step 2: Take a smallest-weight edge $e$ with flag $(e) \neq N O$ among all edges incident to $v$.

Step 3: If $e=e 0, f \operatorname{lag}(e 0)=Y E S$ and return.

Step 4: check_dependency $\left(v^{\prime}, e\right)$, where $v^{\prime}$ is the other endpoint of $e$.

Step 5: If $f \operatorname{lag}(e)=Y E S$, flag $(e 0)=N O$ and return.

Step 6: Go to Step 2.

As we discussed above, we have only to check $|K| / k$ smallest-weight edges. In order to implement it, we need to calculate the weight of the $(|K| / k)$-th smallest edge. There is a theoretically linear-time algorithm [1] for this calculation, but another algorithm shown in [10] is practically more efficient. Once the weight is known, our algorithm is easily modified for the weight.

Next, we analyze the time complexity of this algorithm. Since the algorithm traverses the relation graph in a depth-first fashion, it is clear that the time complexity is linear if sorting in Step 2 of check_dependency can be performed in a constant time. In order to prove it, the following property is useful [4].

Property 4 At most $c_{e}$ edges are in-coming to any node in the nearest neighbor graph, where $c_{e}$ is a constant.

By this property, the number of edges to be sorted in Step 2 of check_dependency is constant. As a result, it is proven that our algorithm has linear time complexity.

Property 5 Our algorithm requires $O(|K|)$ time to find a canonical independent set on the nearest neighbor graph for $K$.

\section{Zero-Skew Routing Algorithm}

Now, we present our linear-time zero-skew routing algorithm. Let $K$ be a set of segments. Initially, $K=S$. Our algorithm has two phases, Find_Center and Embedding. In Find_Center, segments for all internal nodes are calculated in a bottom-up fashion, and then, in Embedding. the best position of each node is determined in a top-down fashion.

The algorithm is stated as follows:

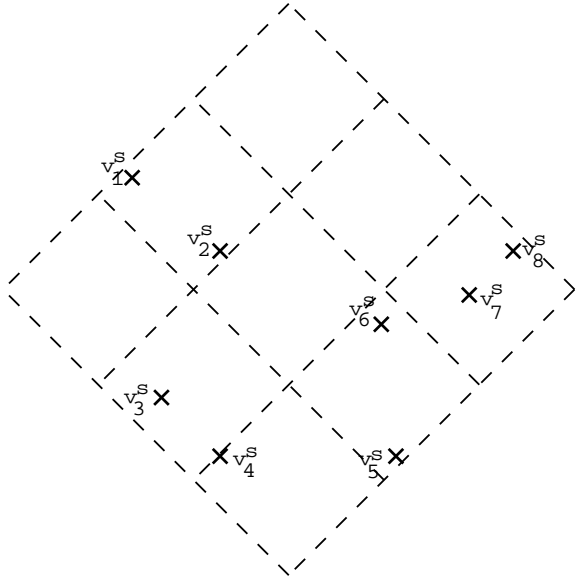

Figure 5: Bucket Decomposition for $K=\left\{v_{1}^{s}, \ldots, v_{8}^{s}\right\}$ in Fig. 4 (a).

\section{Algorithm Find_Center}

Step 1: $K:=S$.

Step 2: If $|K|=1$, stop.

(The element in $K$ is the segment for the center $v_{c}$.)

Step 3: Let $D$ be the diameter of $K$, and let $d=\sqrt{s(2,(1-1 / k)|K|+1,|K|)}-1$.

Construct buckets of size $(D / d) \times(D / d)$ for $K$.

Step 4: Construct a relation graph $G(K, E)$ on $K$.

Step 5: Calculate $i$-th minimum value $v^{*}$ of $w(e)$ among $\forall e \in E$, where $i=s(1,|K| / k,|K|-1)$.

Step 6: Select the canonical independent set from $\{e \in$ $\left.E \mid w(e) \leq v^{*}\right\}$

Step 7: Apply zero-skew merges associated with all edges in the canonical independent set.

Step 8: Go to Step 2.

\section{Algorithm Embedding}

Step 1: Determine the center $v_{c}$ by selecting the nearest point to the root $v_{r}$ on the segment for $v_{c}$. Route from $v_{r}$ to $v_{c}$.

Step 2: local_embedding $\left(v_{c}\right)$

procedure local_embedding $(v)$

Step 1: If $v$ has no child, return.

Step 2: Let $v_{1}, v_{2}$ be the children of $v$. For $i=\{1,2\}$, determine a point $v_{i}$ on the segment for $v_{i}$ so as to satisfy the zero-skew merge equations. Route from $v$ to $v_{i}$.

Step 3: local_embedding $\left(v_{1}\right)$, local_embedding $\left(v_{2}\right)$.

It is clear that the time complexity of Algorithm Embedding is linear. Also, from the analysis in the previous sections. Steps 3-7 in Algorithm Find_Center are performed in $O(|K|)$ time on the average for $K$. Since the degree of nodes in relation graphs is constant (Property 4), the size of the canonical independent set is $\Theta(|K|)$ 
Table 1: Total Delay Time [nsec] for three algorithms.

\begin{tabular}{|l|c||c|c|c|}
\hline & \#pins & part. [2] & clst. [4, 5] & proposed \\
\hline \hline prim1 & 269 & 6.56 & 5.60 & 5.62 \\
\hline prim2 & 603 & 16.99 & 12.77 & 12.84 \\
\hline r1 & 267 & 2.49 & 1.91 & 1.95 \\
\hline r2 & 598 & 5.61 & 4.06 & 4.30 \\
\hline r3 & 862 & 7.71 & 5.40 & 5.57 \\
\hline r4 & 1903 & 18.58 & 11.58 & 11.69 \\
\hline r5 & 3101 & 31.48 & 17.60 & 18.10 \\
\hline
\end{tabular}

Table 2: CPU Time [sec] for three algorithms. (Clustering-based algorithm $[4,5]$ has $O\left(n^{2}\right)$ time complexity because we did not implement Delaunay triangulation algorithm.)

\begin{tabular}{|l|c||c|c|c|}
\hline & \#pins & part. [2] & clst. [4, 5] & proposed \\
\hline \hline prim1 & 269 & 0.42 & 0.90 & 0.25 \\
\hline prim2 & 603 & 1.92 & 5.03 & 0.70 \\
\hline r1 & 267 & 0.18 & 0.87 & 0.27 \\
\hline r2 & 598 & 0.52 & 4.63 & 0.68 \\
\hline r3 & 862 & 0.82 & 9.72 & 1.05 \\
\hline r4 & 1903 & 2.20 & 48.1 & 2.55 \\
\hline r5 & 3101 & 3.77 & 127.6 & 4.08 \\
\hline
\end{tabular}

for $K$. Therefore, after zero-skew merges associated with a canonical independent set for a set $K$, the size of the resultant set is $O(c|K|)$ where $c$ is a constant less than 1 . As a result, we obtain the linearity of our algorithm.

Lemma 1 Our zero-skew routing algorithm requires $O(n)$ time on the average.

\section{Experimental Results}

Now, we show experimental results with benchmark data prim1-prim2 [7] and r1-r5 [11]. In this experiment, we compared our algorithm with the partitioningbased [2] and the clustering-based algorithms [4, 5]. The clustering-based algorithm $[4,5]$ is currently the best algorithm in the total delay time and theoretically the most efficient in the time complexity. However, this algorithm might not be efficient in practice because the algorithm needs to construct Delaunay triangulations for segments on Manhattan distance, for which no practical algorithm has been proposed. On the other hand, the partitioningbased algorithm [2] is the most practical in the sense of execution time, though the total wire length is much longer compared with the clustering-based algorithm $[4,5]$.

In our algorithm, we used the minimum-delay relation function and the parameter $k=4$. The wire-width optimization proposed in [5] was used in the clustering-based algorithm [4, 5] and our algorithm.

Tables 1 and 2 show the total delay time and CPU time for the experiment. The total delay time is estimated by the formula in [5] that was justified using SPICE simulation. CPU time was measured on a 90MIPS RISC workstation. Note that our implementation for the clusteringbased algorithm $[4,5]$ has $O\left(n^{2}\right)$ time complexity because we did not implement the Delaunay triangulation algorithm, though the total delay time on zero-skew routings generated by our program for the algorithm $[4,5]$ is equal to that reported in [5].

It is observed in Table 1 that our algorithm is $10 \%$ $40 \%$ better than the partitioning-based algorithm [2] for the total delay time. Also, it is important to note that Table 2 indicates that the time complexity of our algorithm is linear, while that of the partitioning-based algorithm [2] is a little more than $O(n)$ (theoretically, $O\left(n \log ^{2} n\right)$ ). Although CPU time for the clustering-based algorithm $[4,5]$ shows $O\left(n^{2}\right)$ time complexity, we believe that our algorithm is still much more efficient than $O(n \log n)$ algorithm because the algorithm needs to construct Delaunay triangulations several times. Tables show that our algorithm generates a zero-skew routing for r5 (3101 pins) within 5 seconds with only $3 \%$ increase of the total delay time compared with the best known algorithm $[4,5]$.

\section{Conclusions}

We have proposed a zero-skew routing algorithm with linear time complexity on the average. In order to achieve linearity, relation graphs are constructed using bucketing technique, and independent edge sets are found by a depth first search. This algorithm is much simpler and more efficient than the best known algorithm which uses Delaunay triangulations for segments on Manhattan distance. Experimental results show that our algorithm generates a zero-skew routing for 3000-pin benchmark data within 5 seconds on a 90MIPS RISC workstation.

\section{ACKNOWLEDGMENT}

The author would like to thank A. B. Kahng and K. D. Boese of UCLA and R. S. Tsay of ArcSys Inc. for providing benchmark data, and R. J. Lipton of Princeton University for valuable discussion.

\section{References}

[1] A. V. Aho, J. E. Hopcroft, and J. D. Ullman, The Design and Analysis of Computer Algorithms. Addison-Wesley, 1976.

[2] T. H. Chao, Y. C. Hsu, J. M. Ho, K. D. Boese, and A. B. Kahng, "Zero skew clock routing with minimum wirelength," IEEE Trans. on CAS II, Vol. 39, pp.799-814, 1992.

[3] J. C. Cong, A. B. Kahng, and G. Robins, "Matching-based methods for high-performance clock routing," IEEE Trans. on $C A D$, Vol. 12, pp.1157-1169, 1993.

[4] M. Edahiro, "A clustering-based optimization algorithm in zeroskew routings," Proc. of 30th DAC, pp.612-616, 1993.

[5] M. Edahiro, "Delay minimization for zero-skew routing," Proc. of 1993 ICCAD, pp.563-566, 1993.

[6] M. Edahiro, "Equi-spreading tree in Manhattan distance," unpublished.

[7] M. A. B. Jackson, A. Srinivasan, and E. S. Kuh, "Clock routing for high-performance ICs," Proc. of 27th DAC, pp.573-579, 1990.

[8] Y. M. Li and M. A. Jabri, "A zero-skew clock routing scheme for VLSI circuits," Proc. of 1992 ICCAD, pp.458-463, 1992.

[9] F. P. Preparata and M. I. Shamos, Computational Geometry: An Introduction. Springer-Verlag, 1985.

[10] R. Sedgewick, Algorithms in C. Addison-Wesley, 1990.

[11] R. S. Tsay, "An exact zero-skew clock routing algorithm," IEEE Trans. on CAD, Vol. 12, pp.242-249, 1993. 